\title{
La mise à jour d'avril 2015 de RDA et son impact sur les instructions relatives à la musique : une étude à l'intention des catalogueurs de musique
}

\author{
par Daniel Paradis et Joseph Hafner
}

\section{Link to English version of the article}

\section{Résumé}

La version de RDA Toolkit mise en ligne en avril 2015 comprend des mises à jour majeures du contenu de la version anglaise de RDA, Ressources : description et accès. Plusieurs des révisions incluses dans cette mise à jour modifient des instructions que les catalogueurs de musique doivent appliquer de façon régulière. Cet article examine les plus importantes de ces révisions dans le but d'aider les catalogueurs de musique à mieux comprendre les divers changements qui peuvent affecter le catalogage de la musique. Il examine en particulier l'impact des révisions en ce qui concerne la mention de responsabilité, l'importance matérielle de la musique notée, la durée, les titres privilégiés des œuvres musicales, les abréviations dans les titres de parties d'œuvres musicales ainsi que les points d'accès représentant des expressions musicales. L'article illustre certains des changements en incluant des exemples qui ont été révisés ou ajoutés. Il comprend également un tableau qui compare la structure des instructions de 6.14.2 avant et après la mise à jour d'avril 2015, en raison des nombreux changements dans ce domaine. Bien que cet article s'adresse principalement aux catalogueurs de musique, il peut aussi intéresser les bibliothécaires de musique désireux d'en apprendre davantage sur le catalogage.

\section{Introduction}

Selon le calendrier établi par le Joint Steering Committee for Development of RDA (JSC), la version de RDA Toolkit mise en ligne chaque année au mois d'avril comprend habituellement des révisions majeures du contenu de RDA, Ressources : description et accès. Ces révisions sont le résultat de propositions soumises l'année précédente par les membres du JSC. La version

Daniel Paradis est bibliothécaire au traitement des documents musicaux à la Direction du traitement documentaire des collections patrimoniales de Bibliothèque et Archives nationales du Québec. Il est le président du Comité de catalogage de l'ACBM et est présentement membre du Comité canadien de catalogage, du JSC Music Working Group et du Comité éditorial francophone responsable de la traduction de RDA en français.

Joseph Hafner est vice-doyen aux collections à la Bibliothèque de McGill et exerce actuellement un second mandat à titre de président de la Commission de catalogage de l'AIBM. Il est bibliothécaire et actif dans le domaine du catalogage de la musique depuis 1989. 
publiée en ligne le 14 avril 2015 ne fait pas exception à cette pratique et comporte des révisions qui ont été discutées lors de la réunion du JSC de novembre 2014. Plusieurs des propositions de révision approuvées par le JSC et intégrées dans la version d'avril 2015 modifient des instructions que les catalogueurs de musique doivent appliquer de façon régulière. Cet article examinera les plus importants de ces changements ${ }^{1}$, dans le but $d^{\prime}$ indiquer aux catalogueurs quelles sont les instructions qui ont été révisées ou supprimées et comment ces changements affecteront le catalogage des documents musicaux. Plus précisément, il examinera l'impact des révisions en ce qui concerne :

- la mention de responsabilité;

- l'importance matérielle de la musique notée;

- la durée;

- les titres privilégiés des œuvres musicales;

- les abréviations dans les titres de parties d'œuvres musicales;

- les points d'accès représentant des expressions musicales.

\section{Mention de responsabilité}

RDA définit une mention de responsabilité comme étant « une mention relative à l'identification et/ou la fonction de toute personne, famille ou collectivité responsable de la création, ou ayant contribué à la réalisation du contenu intellectuel ou artistique d'une ressource ". À strictement parler, les mentions identifiant les interprètes de musique devraient entrer dans le champ d'application de cette définition étant donné que les interprètes contribuent au contenu intellectuel ou artistique d'une ressource, qu'ils soient ou non également responsables, en tout ou en partie, de la création de ce contenu. RDA a cependant maintenu la pratique provenant des RCAA2 en vertu de laquelle les mentions identifiant des interprètes ne pouvaient être transcrites comme mentions de responsabilité qu'à la condition que ces interprètes puissent, dans une certaine mesure, être considérés comme des créateurs du contenu. Les mentions identifiant « les interprètes de musique dont la participation est restreinte à la représentation, l'exécution ou l'interprétation » ou « les interprètes, les narrateurs et/ou les présentateurs » devaient être enregistrées dans l'élément « Interprète, narrateur, et/ou présentateur » qui est traité sous 7.23. Les mentions identifiant " les personnes qui ont contribué à la production artistique et/ou technique d'une ressource » étaient également traitées différemment et devaient être enregistrées dans l'élément "Générique artistique et/ou technique », sous 7.24.

1. Les révisions discutées dans cet article proviennent de cinq propositions soumises par le JSC Music Working Group (6JSC/MusicWG/4, 6JSC/MusicWG/5, 6JSC/MusicWG/6, 6JSC/MusicWG/7 et 6JSC/MusicWG/8) et de deux propositions soumises par l'American Library Association (6JSC/ALA/32 et 6JSC/ALA/36). Les propositions et les documents connexes, y compris les réponses à ces propositions produites par les membres du JSC, sont disponibles sur le site du JSC à l'adresse : http://www.rda-jsc.org/working1.html\#constituency. 
Faire des distinctions entre les différents types de mentions de responsabilité n'était cependant pas justifiable sur la base de principes, même si cela pourrait être considéré comme utile pour des raisons pratiques. Pour cette raison, les renvois sous 2.4.1.1 guidant les catalogueurs vers 7.23 et 7.24 ont été supprimés, élargissant ainsi la portée de 2.4 .1 à toutes les mentions de responsabilité. Jusqu'à présent, il était inhabituel d'inclure des noms d'interprètes de musique classique dans les mentions de responsabilité, mais cela va changer avec cette révision. Dans le domaine de la musique populaire, du jazz, etc., la révision rendra les choses plus faciles pour les catalogueurs en éliminant la nécessité de déterminer si le rôle d'un interprète dépasse la simple représentation, exécution ou interprétation. Des exemples ont été ajoutés à 2.4.2.3 (Enregistrement des mentions de responsabilité relatives au titre propre ${ }^{2}$ ) pour inclure des mentions de responsabilité identifiant des interprètes, comme celui-ci:

Bach

Emerson String Quartet

Des exemples ont également été ajoutés sous 2.4.1.7 (Précision du rôle) pour montrer que des mots tels que des noms d'instruments peuvent être ajoutés pour préciser le rôle d'un interprète, comme celui-ci:

Jorja Fleezanis, Ian Swensen [violins]

Title proper: Quintet for strings in B flat major

7.23.1 et 7.24.1 ont été révisées et renvoient maintenant vers 2.4 (Mention de responsabilité) et 2.17.3 (Note on Statement of Responsibility) pour des instructions sur l'enregistrement d'une personne, famille ou collectivité responsable de l'interprétation, de la narration et/ou de la présentation d'une œuvre ou bien des personnes, familles ou collectivités qui ont contribué à la production artistique et/ou technique d'une ressource. Des renvois vers les chapitres 19 et 20 ont également été ajoutés pour des instructions sur l'enregistrement des relations avec ces personnes, familles et collectivités. Les sous-instructions 7.23.1.1-7.23.1.3 et 7.24.1.1-7.24.1.3 ont été supprimées, de même que les entrées du glossaire pour « Interprète, narrateur et/ou présentateur » et « Générique artistique et/ou technique ».

Parce qu'on a estimé que les catalogueurs voudraient encore avoir la possibilité d'enregistrer des noms d'interprètes ou un générique dans une note au lieu de les transcrire comme mentions de responsabilité, un paragraphe a été ajouté à la fin de 2.4.2.3 demandant aux

2. Les titres et les extraits d'instructions sont donnés en français à moins qu'ils n'aient pas encore d'équivalents dans la version française courante de RDA, auquel cas ils sont donnés en anglais. 
catalogueurs de faire une note sur les personnes, familles ou collectivités qui ne sont pas enregistrées dans la mention de responsabilité, si c'est jugé important pour l'identification, l'accès ou la sélection. Toutefois, une mention considérée comme fondamentale pour la mention de responsabilité relative au titre propre doit être transcrite comme une mention de responsabilité et ne peut pas être enregistrée uniquement dans une note.

Le texte de 2.17.3.5 (Other Information Relating to a Statement of Responsibility) a été modifié pour tenir compte des notes sur les interprètes et sur le générique. II précise maintenant que les notes visées par l'instruction peuvent inclure de l'information qui n'est pas enregistrée dans l'élément Mention de responsabilité. II permet également aux catalogueurs d'inclure un mot ou une courte expression si nécessaire pour préciser le rôle d'une personne, famille ou collectivité nommée dans la note. Pour illustrer les cas où une telle note serait utile, des exemples empruntés à l'ancienne instruction 7.23.1.3 ont été ajoutés. L'un d'eux précise la face d'un disque audio sur laquelle figurent les interprètes, alors qu'un autre donne le nom d'un quatuor à cordes ainsi que les noms de ses membres, avec leurs instruments :

Enregistrements par Willie Nelson (face 1), Bob Wills and his Texas Playboys (face 2), Asleep at the Wheel (face 3) et Freddy Fender (face 4)

Budapest String Quartet (J. Roisman et A. Schneider, violons ; B. Kroyt, alto ; M. Schneider, violoncelle)

Il est encore trop tôt pour évaluer l'impact de ces révisions sur les pratiques des catalogueurs. Étant donné la latitude laissée par RDA sur la façon d'enregistrer les mentions de responsabilité, il est à prévoir, en effet, que la communauté des catalogueurs de musique voudra se doter de meilleures pratiques en la matière pour guider les catalogueurs et encourager une certaine uniformité dans les façons de faire. L'impact de la révision sera donc plus ou moins grand selon que ces meilleures pratiques s'éloigneront ou non des pratiques actuelles.

\section{Importance matérielle de la musique notée}

Sous 3.4.3.2 (Enregistrement de l'importance matérielle de la musique notée), l'exception sur les ressources constituées d'une partition et d'une ou plusieurs parties en une seule unité physique a été élargie pour traiter également des ressources constituées de plusieurs parties en une seule unité physique. Les exemples suivants ont été ajoutés pour illustrer les instructions :

1 score and 2 parts ( 1 volume (unpaged)) 
3 parts $(5,5,5$ pages)

Parts printed in 1 volume with duplicate pagings

20 parts (approximately 100 pages)

Un paragraphe a été ajouté, qui demande de faire une note pour expliquer l'importance matérielle si c'est jugé important pour l'identification ou la sélection et renvoie vers 3.21.2.5.

La portée de 3.21.2.5 a été à son tour étendue. Son libellé a été simplifié et de nouveaux exemples ajoutés. L'instruction se lit maintenant comme suit :

\section{Score and One or More Parts, or Multiple Parts in a Single Physical Unit}

Make a note giving the number and types of units included in a single physical unit if considered important for identification or selection.

\section{EXAMPLE}

4 parts in 1 volume

1 score and 2 parts in 1 volume; parts printed on leaves 8-10

\section{Durée}

Les instructions sur la durée sous 7.22 ont subi une importante révision. La distinction qui existait auparavant entre la durée de lecture, de projection, etc. pour les ressources audio et vidéo et la durée d'exécution pour la musique notée n'existe plus et les instructions ont été généralisées pour s'appliquer aux deux types de durée. En conséquence, 7.22.1.4 (Durée d'exécution) a été supprimée et la définition de durée sous 7.22.1.1 a été modifiée afin de refléter la nouvelle organisation. Elle dit maintenant que la durée est la durée de lecture, de projection, d'exécution, etc. du contenu de la ressource. II convient de noter que la durée d'exécution n'est plus limitée à la musique notée. Comme certains des nouveaux exemples l'illustrent, elle s'étend maintenant aux autres types de contenu noté qui comportent une durée, par exemple les pièces de théâtre, les monologues, les chorégraphies, etc.

7.22.1.3 a été renommée "Recording Duration » et permet désormais une plus grande souplesse en disant d'enregistrer la durée dans la forme préférée par l'agence créant des données ". Cela laisse par exemple le choix à une agence d'utiliser des symboles (par exemple, " $3 \mathrm{~min}, 23 \mathrm{~s}$ »), des termes (qui, en anglais, doivent alors être abrégés selon les instructions de 
l'annexe B, par exemple, « 40 min. ») ou de chiffres séparés par des deux-points (par exemple, « 02:30:04»).

Les méthodes permises pour enregistrer la durée totale ont été simplifiées et ne donnent plus la préférence à la durée qui figure sur la ressource. La méthode privilégiée consiste à enregistrer la durée exacte si celle-ci peut être facilement établie, qu'elle figure ou sur la ressource ou non. Lorsque la durée de lecture est mentionnée sur la ressource, mais diffère considérablement de la durée réelle, les catalogueurs n'ont plus la possibilité d'enregistrer la durée mentionnée suivie de c'est-à-dire et de la durée réelle. Il faut désormais enregistrer la durée exacte et donner la durée qui figure sur la ressource dans les particularités de la durée, si c'est jugé important pour l'identification ou la sélection. Si la durée exacte ne peut pas être facilement établie, on enregistre une durée approximative qui figure comme telle sur la ressource ou peut être facilement estimée, précédée de environ. La durée est omise si elle ne peut être facilement déterminée ou estimée. L'alternative permettant de donner la durée de lecture, de projection, etc. uniforme, de chaque unité dans une ressource en plusieurs parties suivie par chacun ou chacune a été supprimée. Cette information peut toutefois encore être enregistrée dans les particularités de la durée si elle est jugée utile.

L'instruction sur la durée des parties individuelles, auparavant sous 7.22.1.5, a été déplacée sous 7.22.1.4 et s'intitule désormais « Duration of Component Parts » pour préciser qu'elle s'applique aux unités de contenu intellectuel, comme les chansons sur un disque compact ou les actes d'une pièce de théâtre, et non à des unités physiques. Une alternative a été ajoutée qui permet d'enregistrer la durée totale de la ressource au lieu ou en plus de la durée des parties composantes.

Une nouvelle instruction sur les particularités de la durée a été ajoutée sous 7.22.1.5, avec des exemples comme ceux-ci :

16:00 per audio cylinder

Duration of each cylinder in a set of $\mathbf{3 1}$ audio cylinders

Running time given as 155 min. on container

Duration stated on resource that has an actual duration of $113 \mathrm{~min}$.

Enfin, 7.22.1.6 (Ressource contenant du son et/ou des images animées et du texte, des images fixes, etc.) a été supprimée. 


\section{Titres privilégiés des œuvres musicales}

Les instructions sur le choix et l'enregistrement des titres privilégiés des œuvres musicales sous 6.14.2 ont fait l'objet d'une importante révision pour les présenter dans une structure plus logique et pour faire en sorte que leur libellé soit en autant que possible le même que celui de 6.2.2 $2^{3}$. Tout d'abord, 6.14.2.2 (Sources d'information) a été modifiée pour y effectuer des modifications similaires à celles faites sous l'instruction générale 6.2.2.2 sur les sources d'information relatives au titre privilégié de l'œuvre. Les instructions existantes, qui spécifiaient les sources à utiliser selon que l'œuvre avait été créée après 1500 ou avant 1501, ont donc été remplacées par des instructions générales s'appliquant à toutes les œuvres, peu importe l'époque de leur création. D’après ces instructions, on doit déterminer le titre privilégié d'une œuvre musicale à partir des ressources matérialisant l'œuvre ou à partir des sources de référence. Une phrase a été ajoutée disant d'appliquer 6.14.2.3 lors du choix de la source d'information. Les instructions existantes n'ont pas disparu pour autant mais ont été incorporées dans les nouvelles instructions 6.14.2.3.1 et 6.14.2.3.2.

6.14.2.3 (Choix du titre privilégié d'une œuvre musicale) a maintenant une physionomie complètement différente. L'instruction était auparavant constituée d'une règle s'appliquant aux œuvres de toutes les époques et disant de choisir « le titre original du compositeur dans la langue de sa rédaction ». La règle était ensuite suivie de trois exceptions : "Titre plus connu dans la même langue ", "Titres longs » et "Série numérotée ». 6.14.2.3 prend maintenant l'aspect d'une règle générale renvoyant vers deux sous-instructions traitant des œuvres créées après 1500 (6.14.2.3.1) et des œuvres créées avant 1501 (6.14.2.3.2). Cette révision fait en sorte que 6.14.2.3 adopte la même structure que les instructions générales 6.2.2.3-6.2.2.5. C'est donc désormais sous 6.14.2.3.1-6.14.2.3.2 que l'on retrouve les instructions spécifiques relatives aux sources d'information qui étaient auparavant sous 6.14.2.2 et qui ont été réécrites en prenant pour modèle le libellé de 6.2.2.4-6.2.2.5 (" choose as the preferred title the title or form of title in the original language by which the work is commonly identified ... »). Cette révision a pour conséquence de mieux respecter le principe de représentativité en donnant explicitement la priorité à un titre (ou à une forme de titre) sous lequel l'œuvre est couramment identifiée plutôt qu'au titre original du compositeur.

Malgré les apparences, cela ne représente pas un changement majeur par rapport aux instructions antérieures puisque la première exception sous 6.14.2.3 demandait de choisir comme titre privilégié un autre titre dans la même langue sous lequel l'œuvre est plus connue plutôt que le titre original du compositeur. L'exception demandant de choisir un titre court par

3. Pour une comparaison de la structure des instructions de 6.14.2 avant et après la mise à jour du 14 avril, voir le Tableau 1 à la fin de cet article. 
lequel l'œuvre est communément identifiée dans les sources de référence privilégiait, elle aussi, un titre sous lequel l'œuvre est mieux connue. La révision a l'avantage d'uniformiser les instructions en reformulant l'ancienne instruction 6.14.2.3 dans les mêmes termes que les instructions générales 6.2.2.4-6.2.2.5et, en faisant de l'exception la règle, de mettre en évidence le principe de représentativité.

Pour prévoir les cas où il ne serait pas possible de choisir comme titre privilégié le titre ou la forme de titre dans la langue originale sous laquelle l'œuvre est couramment identifiée, une instruction indiquant de choisir comme titre privilégié le titre original du compositeur ou le titre propre de l'édition originale, dans cet ordre de préférence, a été ajoutée sous 6.14.2.3.1. Cette instruction doit être suivie lorsqu'il n'y a pas de titre (ou de forme de titre) dans la langue employée à l'origine par le compositeur sous lequel l'œuvre est couramment identifiée, lorsque la langue du titre employée à l'origine par le compositeur ne peut être établie de même qu'en cas de doute.

Suite à ces changements, les exceptions demandant de choisir un titre dans la même langue sous lequel l'œuvre est plus connue ou un titre court par lequel l'œuvre est communément identifiée dans les sources de référence sont devenues redondantes et ont été supprimées. L'exception permettant de choisir un titre court forgé par le catalogueur plutôt qu'un titre très long a été jugée incompatible avec le principe de représentativité et a elle aussi été supprimée. L'exception concernant les séries d'œuvres numérotées a été quant à elle déplacée sous 6.14.2.5 (Recording the Preferred Title for an Individual Musical Work) puisqu'elle a trait non pas au choix du titre privilégié mais à son enregistrement.

Pour tenir compte du fait que des instructions particulières ont été ajoutées sous 6.2.2 pour traiter, d'une part, des cas où un titre dans la langue originale n'est pas trouvé ou n'est pas applicable (6.2.2.6) et, d'autre part, des cas où un titre est trouvé dans une écriture non privilégiée (6.2.2.7), les renvois pertinents vers ces instructions ont été incorporés à la fois sous 6.14.2.3.1 et 6.14.2.3.2.

6.14.2.4-6.14.2.5 ont été révisées pour combler diverses lacunes. 6.14.2.4 se présentait comme une règle générale sur l'enregistrement des titres privilégiés mais était en fait conçue pour ne s'appliquer qu'aux œuvres individuelles. L'instruction a été réécrite pour avoir une portée véritablement générale et mentionne maintenant explicitement qu'elle s'applique autant aux œuvres individuelles qu'aux compilations d'œuvres. On y a aussi incorporé une règle demandant de ne pas enregistrer un titre alternatif comme partie du titre privilégié, calquée sur la règle générale équivalente sous 6.2.2.8 (auparavant sous 6.2.2.4). Le contenu concernant les éléments à omettre du titre a été quant à lui déplacé sous 6.14.2.5.1. 
À 6.14.2.5, on trouve maintenant une nouvelle instruction qui traite de l'enregistrement du titre privilégié d'une œuvre musicale individuelle et renvoie à l'instruction générale 6.14.2.4. L'exception auparavant à 6.14.2.3 sur les séries d'œuvres numérotées y a été déplacée et, pour plus de clarté, a été reformulée avec trois conditions plutôt que deux. Les instructions sur les omissions (6.14.2.5.1, auparavant 6.14.2.4) ainsi que celles sur les titre privilégiés constitués uniquement du nom d'un type de composition (6.14.2.5.2, auparavant 6.14.2.5) se présentent désormais comme des sous-instructions de 6.14.2.5, pour clarifier qu'elles s'appliquent uniquement aux œuvres individuelles et non aux compilations.

On remarquera deux modifications qui affectent les éléments à omettre dans le titre privilégié donnés sous 6.14.2.5.1. Le libellé du paragraphe d) spécifie maintenant que les nombres dont il est question sont les nombres cardinaux et ordinaux (cardinal and ordinal numbers), pour bien distinguer ce paragraphe du paragraphe précédent (serial, opus, and thematic numbers). Le paragraphe f) auparavant sous 6.14.2.4 et disant d'omettre « les adjectifs et épithètes qui ne font pas partie du titre original de l'œuvre " a quant à lui été supprimé car de tels termes ne devraient logiquement pas faire partie du titre privilégié choisi selon 6.14.2.3. Les exemples illustrant les omissions sous 6.14.2.5.1 ont été revus afin de les rendre plus conformes aux principes éditoriaux suivis dans RDA. Des notes ont entre autres été ajoutées pour expliquer l'application de l'instruction ainsi que ses exceptions, comme dans les exemples suivants :

Präludien und Fugen

Preferred title before omissions: Sechs Präludien und Fugen für Organ. Number and statement of medium of performance omitted

The seventh trumpet

\section{Number recorded because it is an integral part of the title}

Pour plus de clarté, les contrexemples ont été regroupés à la fin et sont clairement identifiés comme tels par la préposition but qui les précède.

On notera deux révisions touchant les sous-instructions de 6.14.2.5.2. Sous 6.14.2.5.2.1, l'exception sur les œuvres désignées par des titres tels qu'étude, fantasia ou sinfonia concertante ou des termes apparentés n'indique plus qu'elle s'applique uniquement aux œuvres destinées à l'exécution de concert. Cette distinction n'était plus jugée nécessaire ni pertinente et a été éliminée. Sous 6.14.2.5.2.2, les notes accompagnant les exemples ont été réécrites pour récapituler et rendre plus explicites les différentes étapes à travers lesquelles un titre a pu passer pour en arriver à la forme finalement enregistrée, ce qui donne par exemple : 
Quartet

Preferred title before omissions: Quatuor pour 2 hautbois et 2 bassons. Title after omissions: Quatuor. English language form recorded by an English-language agency in Canada because it is a cognate to the French title

Finalement, on prendra note que l'instruction 6.14.2.6 (Duos) a été supprimée. Outre qu'il était difficile à justifier dans un code qui se veut international, l'emploi normalisé du terme Duet pour les œuvres diversement intitulées duos, duets, etc. ne respectait pas le principe de représentativité selon lequel la forme du titre choisie comme titre privilégié devrait être celle qui est le plus couramment utilisée pour identifier l'œuvre.

\section{Abréviations dans les titres de parties d'ouvres musicales}

Les instructions 6.2.1.9 et B.3, qui régissent l'utilisation des abréviations dans les titres d'œuvres, ont été modifiées pour permettre l'abréviation du mot Numéro ou son équivalent dans une autre langue. Le mot Numéro ou son équivalent peuvent maintenant être abrégés, mais uniquement lorsqu'ils apparaissent dans le titre d'une partie d'une œuvre musicale et lorsque ce mot précède un numéro utilisé pour identifier cette partie. L'exemple suivant a été ajouté pour illustrer cette situation :

\section{Nr. 32, Sheherazade}

\section{Title for a part of a musical work}

Cette pratique était celle suivie par les catalogueurs américains lorsque les RCAA2 étaient en vigueur et est dérivée de l'interprétation émise par la Bibliothèque du Congrès pour la règle 25.32A1. Le JSC a jugé utile d'intégrer cette pratique dans RDA car elle continue de répondre à un besoin. L'interprétation de la Bibliothèque du Congrès disait également comment enregistrer un numéro qui identifie une partie d'une œuvre musicale et quoi faire lorsque ce numéro n'est pas précédé d'un terme comme Numéro. Ces directives ont été incorporées sous 6.14.2.7.1 (Une partie) dans un nouveau paragraphe qui dit ceci :

Record a number used to identify the part as a numeral. If the number of the part has no general term associated with it, precede the number with the abbreviation of Number or its equivalent in another language (see appendix B (B.3)). Record the abbreviation in the language in which the preferred title of the work as a whole is recorded. 


\section{Points d'accès représentant des expressions musicales}

La révision de 6.28.3 (renommée Constructing Authorized Access Points Representing Musical Expressions) visait principalement à élargir la portée de l'instruction, laquelle était limitée aux types d'expressions musicales mentionnées sous 6.28.3.1, à savoir :
a) arrangements, transcriptions, etc.;
b) accompagnements, etc. ajoutés;
c) esquisses;
d) partitions de chant et partitions de chœur;
e) traductions.

Pour les autres types d'expressions musicales, RDA disait d'appliquer les instructions générales sous 6.27.3 (Point d'accès autorisé représentant une expression). Celles-ci permettent d'inclure le type de contenu, la date de l'expression, la langue de l'expression et/ou une autre caractéristique distinctive de l'expression dans un point d'accès représentant une expression particulière d'une œuvre ou d'une ou plusieurs parties d'une œuvre. II n'existait cependant aucune instruction qui aurait permis de recourir à l'un ou l'autre de ces éléments dans le cas des expressions musicales traitées sous 6.28.3.1. En outre, l'instruction n'avait pas prévu le cas où une expression aurait appartenu à plus d'un des types qu'elle visait expressément (par exemple, une partition de chant qui serait aussi une traduction). 6.28.3 a donc été révisée pour lever ces restrictions et autoriser l'ajout d'un ou plusieurs des éléments suivants :
a) le type de contenu;
b) la date de l'expression;
c) la langue de l'expression;
d) une autre caractéristique distinctive de l'expression d'une œuvre musicale.

L'instruction a également été considérablement simplifiée pour réduire les redondances tant à l'intérieur de l'instruction elle-même qu'avec les autres instructions, principalement 6.18. Elle est maintenant réduite à une seule instruction, calquée sur 6.27.3, qui liste les éléments qu'il est possible de combiner avec le point d'accès autorisé représentant l'œuvre ou la ou les parties d'une œuvre pour construire un point d'accès représentant une expression particulière d'une œuvre ou d'une ou plusieurs parties d'une œuvre. Des renvois guident désormais le catalogueur vers les instructions qui traitent des éléments spécifiques $(6.9,6.10,6.11$ et 6.18$)$ pour de plus amples directives sur la façon d'enregistrer ces éléments. Un renvoi approprié a aussi été ajouté à la fin de 6.27.3 pour diriger les catalogueurs vers 6.28.3.

Cette révision a suscité à son tour des modifications à 6.18 (Autre caractéristique distinctive de l'expression d'une œuvre musicale). La définition de l'élément a été élargie pour permettre l'enregistrement d'attributs autres que les termes arrangé, Esquisses, Partition de chant, 
Partitions de chant, Partition de chœur ou Partitions de chœur. La nouvelle définition, dont la formulation est empruntée à celle employée à 6.12.1.1 pour l'élément Autre caractéristique distinctive de l'expression, spécifie maintenant qu'il s'agit d'une caractéristique autre que le type de contenu, la langue de l'expression ou la date de l'expression. Elle devrait par conséquent offrir aux catalogueurs une plus grande souplesse lors de l'enregistrement des attributs des expressions musicales.

Les instructions concernant les arrangements, transcriptions, etc. ont également été révisées. 6.18.1.4 a été réécrite pour prendre l'aspect d'une règle générale s'appliquant à tous les arrangements, etc., et non plus uniquement à la catégorie de la musique dite " sérieuse ", " classique » ou " savante ». Les paragraphes énonçant les catégories d'expressions dont traite l'instruction ont été reformulés pour se conformer à la définition d'arrangement dans le glossaire. L'instruction s'applique désormais lorsqu'une expression résulte d'un changement dans la distribution d'exécution ou bien d'une simplification ou d'une autre modification de l'œuvre, avec ou sans changement dans la distribution d'exécution. L'instruction sur les arrangements, etc. dans un genre " populaire ", auparavant sous 6.18.1.4.1, est traitée maintenant comme une exception à l'instruction générale. Comme l'instruction sur les accompagnements, etc. ajoutés, auparavant située sous 6.28.3.3, n'avait pas d'équivalent sous 6.18, une seconde exception a été ajoutée sous 6.18.1.4 pour demander ne pas enregistrer arrangé si un accompagnement instrumental ou des parties supplémentaires ont été ajoutés à une œuvre ou à une ou plusieurs parties d'une œuvre et que la musique originale n'a pas été altérée.

\section{Conclusion}

Les révisions étudiées dans cet article s'inscrivent dans le cadre des efforts continus déployés par le JSC en vue de mieux adapter les instructions héritées des RCAA2 au contexte de RDA - en particulier celles relatives à la musique - de résoudre les incohérences observées entre les instructions et de combler les éventuelles lacunes. Toutes ces révisions n'auront pas un impact égal ou nécessairement visible dans les notices bibliographiques; certains changements seront plus susceptibles que d'autres d'être remarqués et, espérons-le, d'être appréciés par les usagers du catalogue. Les révisions visant à simplifier les instructions ou à en améliorer la structure plutôt qu'à faire en sorte que leur application produise des résultats différents n'en sont toutefois pas moins importantes car elles contribueront à faciliter l'apprentissage de RDA ainsi que son application. 


\section{TABLEAU 1}

\section{Comparaison de la structure des instructions de 6.14.2 avant et après la mise à jour du 14 avril 2015}

Note: Les sous-instructions de 6.14.2.7 n'ont pas été affectées par la réorganisation de 6.14.2 et n'ont pas été incluses dans le tableau. Les titres d'instructions sont donnés uniquement en anglais s'ils n'ont pas encore d'équivalents dans la version française courante de RDA.

\begin{tabular}{|c|c|c|}
\hline Structure avant la mise à jour & \multicolumn{2}{|r|}{ Structure après la mise à jour } \\
\hline 6.14.2 Titre privilégié d'une œuvre musicale & 6.14 .2 & Titre privilégié d'une œuvre musicale \\
\hline 6.14.2.1 Champ d'application & 6.14.2.1 & Champ d'application \\
\hline 6.14.2.2 Sources d'information & 6.14.2.2 & Sources d'information \\
\hline $\begin{array}{l}\text { 6.14.2.3 Choix du titre privilégié d'une œuvre } \\
\text { musicale }\end{array}$ & 6.14 .2 .3 & $\begin{array}{l}\text { Choix du titre privilégié d'une œuvre } \\
\text { musicale }\end{array}$ \\
\hline & 6.14.2.3.1 & Musical Works Created after 1500 \\
\hline & 6.14.2.3.2 & Musical Works Created Before 1501 \\
\hline $\begin{array}{l}\text { 6.14.2.4 Enregistrement du titre privilégié d'une } \\
\text { œuvre musicale }\end{array}$ & 6.14.2.4 & $\begin{array}{l}\text { Enregistrement du titre } \\
\text { privilégié d'une œuvre musicale }\end{array}$ \\
\hline & 6.14 .2 .5 & $\begin{array}{l}\text { Recording the Preferred Title for an } \\
\text { Individual Musical Work }\end{array}$ \\
\hline & 6.14.2.5.1 & Omissions \\
\hline $\begin{array}{l}\text { 6.14.2.5 Titre privilégié constitué uniquement du } \\
\text { nom d'un type de composition }\end{array}$ & 6.14 .2 .5 .2 & $\begin{array}{l}\text { Titre privilégié constitué uniquement } \\
\text { du nom d'un type de composition }\end{array}$ \\
\hline 6.14.2.5.1 Choice of Language & 6.14.2.5.2.1 & Choice of Language \\
\hline 6.14.2.5.2 Singular or Plural Form & 6.14.2.5.2.2 & Singular or Plural Form \\
\hline 6.14.2.6 Duos & 6.14.2.6 & Duos [supprimée] \\
\hline $\begin{array}{l}\text { 6.14.2.7 Enregistrement du titre privilégié d'une } \\
\text { ou plusieurs parties d'une œuvre } \\
\text { musicale }\end{array}$ & 6.14.2.7 & $\begin{array}{l}\text { Enregistrement du titre privilégié } \\
\text { d'une ou plusieurs parties d'une } \\
\text { œuvre musicale }\end{array}$ \\
\hline 6.14.2.8 Compilations d'œuvres musicales & 6.14.2.8 & Recording the Preferred Title for a \\
\hline $\begin{array}{ll}\text { 6.14.2.8.1 Enregistrement du titre privilégié des } \\
\text { d'œuvres musicales }\end{array}$ & & $\begin{array}{l}\text { Compilation of Musical Works by One } \\
\text { Composer }\end{array}$ \\
\hline 6.14.2.8.2 CEuvres complètes & 6.14.2.8.1 & CEuvres complètes \\
\hline $\begin{array}{l}\text { 6.14.2.8.3 Complete Works for One Broad } \\
\text { Medium }\end{array}$ & 6.14.2.8.2 & $\begin{array}{l}\text { Complete Works for One Broad or } \\
\text { Specific Medium }\end{array}$ \\
\hline $\begin{array}{ll}\text { 6.14.2.8.4 Complete Works for One Specific } \\
\text { Medium }\end{array}$ & & \\
\hline $\begin{array}{ll}\text { 6.14.2.8.5 Complete Works of One Type for One } \\
\text { Specific Medium or Various Media }\end{array}$ & 6.14 .2 .8 .3 & $\begin{array}{l}\text { Complete Works of a Single Type of } \\
\text { Composition for One Specific } \\
\text { Medium or Various Media }\end{array}$ \\
\hline 6.14.2.8.6 Compilations incomplètes & 6.14.2.8.4 & Compilations incomplètes \\
\hline
\end{tabular}

